\title{
A VARIATIONAL APPROACH TO STATISTICALLY NONHOMOGENEOUS FIELDS*
}

\author{
BY \\ A. SOMOROFF \\ Naval Air Development Center, Johnsville, Warminster, Pennsylvania
}

\begin{abstract}
Classical variational principles of elasticity are cast in a form amenable to the treatment of certain problems concerned with statistically nonhomogeneous fields. The variational principles are utilized to obtain bounds directly upon the ensemble average values of physical quantities which in some sense characterize their problems. General formulations and applications are given for randomly heterogeneous elastic media and elastically supported beams with random axial variation of sectional rigidity and foundation stiffness. Bounds are obtained for the average displacement at the inner surface of a hollow sphere under pressure, the average elongation of a cylinder in uniform tension, and the average displacement of a beam directly under a point load. General methods are given for the selection of the deterministic admissible functions which provide the best bounds that can be obtained from classical principles for this restricted class of admissible functions. The closeness of the bounds is of the same order as the elementary bounds on effective material properties, which are of quantitative value only for small dispersion of the random coefficients. In a special statistically homogeneous case, previously obtained bounds for an effective Young's modulus are regained. Usually, however, the bounded quantities do not possess the generality of effective properties but are more closely associated with the results of particular problems. The developments of the present paper are considered an initial approach to the investigation of statistically nonhomogeneous problems.
\end{abstract}

1. Introduction. Although considerable work has been done on randomly heterogeneous materials, the major portion of this work has been concerned with the investigation of effective properties. Incomplete information and mathematical complexities preclude a direct solution to the boundary value problems. Thus statistical concepts have been useful in material description. The distribution of properties within the heterogeneous media has usually been considered as statistically homogeneous and isotropic. In addition almost all preceding investigations have treated statistically homogeneous physical fields.

Statistical homogeneity requires that ensemble average quantities be independent of absolute position within the media. For these special circumstances an ergodic-type hypot -sis may permit the interchange of ensemble average and spatial average quantities. Previous treatments concerned with statistically homogeneous problems were, therefore, often able to work with spatial average quantities, which arose rather naturally in the course of development. Local spatial average quantities are related by effective

* Received March 4, 1969; revised version received June 5, 1969. 
constitutive properties in the same manner as uniform moduli relate quantities in homogeneous media.

We note that the region over which the local spatial averaging procedure is carried out must be large compared to the size of material inhomogeneities. However if a problem is not homogeneous in a statistical sense, and ensemble average fields vary rapidly in distances comparable to a characteristic length of the material inhomogeneities, then the spatial average quantities lose much of their significance. It is not clear that the concept of an effective property is meaningful when such conditions exist.

Another circumstance in which it may be difficult to use effective properties is the occurrence of nonconstitutive random parameters. Suitable relations for the definition of effective properties, as are conveniently provided by the constitutive equations for constitutive coefficients, are difficult to envision for these parameters.

The present paper investigates problems for which fields need not be statistically homogeneous. In general it can be shown that for fields governed by differential equations with random coefficients, a solution for simply the average value of the dependent variable requires complete statistical knowledge of the random coefficients. Because this statistical information is difficult to obtain, results are often in the form of bounds which provide limited knowledge based upon a limited input. The use of variational principles to bound effective properties for heterogeneous materials is well established. Paul [1] used classical variational principles of elasticity to bound effective elastic moduli. Brown [2] improved bounds of this nature with the use of a perturbation series to input additional statistical information, as did Beran [3] who recast the standard elasticity principles in terms of ensemble averages. Improvements upon the original bounds were also obtained by Hashin and Shtrikman [4] with a variational principle formulated in terms of polarization variables.

It is the purpose of the present paper to examine several areas for which the concept of effective properties is not clearly applicable. In particular, variational principles of a classical nature are utilized for the derivation of bounds on quantities which, in some sense, characterize their respective problems. Without reference to an ergodic hypothesis, variational principles are cast in a form which of ten permits the ensemble average value of an interesting physical quantity to be isolated and subsequently bounded. The bounds are derived in terms of one point average values of the relevant random coefficients or their inverse. In general it is shown that a knowledge of the one point averages of the random coefficients is sufficient to obtain bounds from classical variational principles only for deterministic admissible ficlds. In addition, a consistent approach is given for the selection of the particular deterministic admissible fields which provide the best bounds that can be obtained from classical variational principles, for this restricted class of admissible functions. The closeness of the upper and lower bounds is of the same order as for the elementary bounds on effective properties.

In the first sections we consider randomly heterogeneous elastic media. The results of a general development are applied to the derivation of bounds on the average radial displacement of the inner surface of a hollow sphere under pressure. Another application provide, inunds on the elongation of a cylinder acted upon by uniform axial forces.

In the following sections we are concerned with elastically supported beams for which the flexural rigility and foundation modulus may vary in a random manner along the beam length. An illustration is given in which bounds are derived for the average deflection of a simply supported beam directly under a concentrated force. 
All developments which follow are explicitly presented for continuous random parameters. But with minor modifications in the derivation of the extremum principles, the final results remain valid for piecewise continuous random parameters.

2. Heterogeneous elastic media. We shall consider several problems governed by the equations of linear elasticity. For an isotropic medium with spatially varying elastic coefficients Hooke's law may be written as

$$
\sigma_{i j}(\vec{x})=2 G(\vec{x}) \epsilon_{i j}(\vec{x})+\left(k(\vec{x})-\frac{2}{3} G(\vec{x})\right) \epsilon_{l l}(\vec{x}) \delta_{i j}
$$

where

$\sigma_{i j}$ is the symmetric stress tensor,

$\boldsymbol{\epsilon}_{i j}$ is the symmetric strain tensor,

$G(\vec{x})$ is the shear modulus and $k(\vec{x})$ the bulk modulus.

Although the medium is required to be locally isotropic, we allow that it may be randomly heterogeneous. The elastic coefficients of this medium are then random functions of position which can be written as

$$
\begin{aligned}
& G(x)=\overline{G(x)}+G^{\prime}(x), \\
& k(x)=\overline{k(x)}+k^{\prime}(x) .
\end{aligned}
$$

The overbar indicates an average in an ensemble sense. Primes denote fluctuating components which have zero mean.

If the distribution of material properties is homogeneous in a gross or statistical sense, then $\bar{G}$ and $\bar{k}$ are independent of absolute position. Statistical homogeneity is a concept most usually applied to media of infinite extent. In the present work, however, bodies of finite extent are considered. It is obvious, therefore, that in the present context, statistical homogeneity of random coefficients implies statistical homogeneity only within the regions of the given bodies. We note that a statistically homogeneous distribution of material coefficients need not be assumed for the general developments of this paper, although it is sometimes necessary for the calculation of final results in particular problems.

The random nature of the material coefficients does not explicitly alter the form of the governing equations. Relations between the strain tensor and displacements are given by

$$
\epsilon_{i j}=\frac{1}{2}\left(\frac{\partial u_{i}}{\partial x_{i}}+\frac{\partial u_{i}}{\partial x_{i}}\right) \text {. }
$$

The equilibrium equations are

$$
\partial \sigma_{i j} / \partial x_{i}+F_{i}=0
$$

where the components of body force per unit volume, $F i$, are assumed to be deterministic.

Combining (1), (2), and (3), the equilibrium equations may be expressed in terms of displacements as

$$
2 \frac{\partial}{\partial x_{i}}\left[G\left(\frac{\partial u_{i}}{\partial x_{i}}+\frac{\partial u_{i}}{\partial x_{i}}\right)\right]+\frac{\partial}{\partial x_{i}}\left[\left(k-\frac{2}{3} G\right) \frac{\partial u_{l}}{\partial x_{l}}\right]+F_{i}=0 .
$$

We assume the elastic medium to occupy a region $R$ bounded by a surface $S$. Displacements are prescribed on the portion of the surface $S_{u}$, while tractions, given 
by $T_{i}=\sigma_{i i} n_{i}$, are prescribed on the portion of the surface $S_{T}$. The $n_{i}$ are components of the unit outer normal on $S$.

The above equations, along with compatibility relations, define the general problem with which we are concerned.

VARIational PRINCIPlaEs OF Elasticity-Minimum potential Energy. The functional

$$
V_{D}=\frac{1}{2} \int_{R} \sigma_{i j} \epsilon_{i j} d R-\int_{R} F_{i} u_{i} d R-\int_{S T} T_{i} u_{i} d S
$$

which may be expressed in terms of displacements as

$$
\begin{aligned}
V_{p}=\frac{1}{2} \int_{R}\left\{\frac { G } { 2 } \left[\left(\frac{\partial u_{i}}{\partial x_{j}}\right)^{2}+\left(\frac{\partial u_{i}}{\partial x_{i}}\right)^{2}\right.\right. & \left.+2 \frac{\partial u_{i}}{\partial x_{i}} \frac{\partial u_{i}}{\partial x_{i}}\right] \\
& \left.+\left(k-\frac{2}{3} G\right) \frac{\partial u_{l}}{\partial x_{l}} \frac{\partial u_{m}}{\partial x_{m}}-2 F_{i} u_{i}\right\} d R-\int_{S T} T_{i} u_{i} d S
\end{aligned}
$$

is stationary and attains its absolute minimum value for that admissible displacement ficld which corresponds to the equilibrium state [5].

Admissible displacement fields are required to be continuous and satisfy displacement boundary conditions. The displacements of the equilibrium state also satisfy Eqs. (4), and yield stresses which satisfy the traction conditions on $S_{T}$.

If the extremum value of the potential energy associated with the equilibrium displacements is denoted by $V_{p}$, and the functional corresponding to any admissible displacement field, $u_{i}^{0}$, is denoted by $\mathrm{V}_{p}^{\circ}$, then we have the following inequality:

$$
r_{p} \leq V_{p}^{\circ} .
$$

We wish to use the ine(puality of (7) and another inequality provided by the complementary energy principle to obtain upper and lower bounds on quantities which characterize particular problems. In those problems for which fields are statistically homogeneous, effective material properties may conveniently provide this characterization. The present formulation, however, must be applicable to nonhomogeneous problems, and be suited to the derivation of bounds on quantitics other than effective properties. These quantities do not possess the generality of effective properties but are more usually associated with specific germetries and conditions.

The nature of problems: which can be treated is exemplified by the derivation of bounds on the average radial displacement at the inner surface of a randomly heterogeneous hollow elastic sphero. A uniform pressure, $p_{A}$, is assumed to act on the inner surface of radius $A$. The tractions on the outer surface of radius $B$ and the body forces throughout the sphere are taken as identically zero. It is clear that for these conditions the stress and strain fields will not be statistically homogeneous, since even in a uniform body there womk be radial variation of the ficlds.

While the notation used in the goncral formulation is adequate to describe this problem, a spherical cocirdinate system is more natural for a specific treatment. In spherical coordinates the conditions specified above can be expressed as follows: 
(a) $T_{r}=p_{A}, T_{\theta}=T_{\phi}=0, \quad$ on the inner surface of radius $A$

(b) $T_{r}=T_{\theta}=T_{\phi}=0, \quad$ on the outer surface of radius $B$

where $r, \theta$, and $\phi$ are the usual coordinates of a spherical system.

Even with these relatively simple conditions the expression for $V_{p}$ as given in (6), is not appreciably reduced in complexity. As the general development progresses, we will consider this heterogeneous sphere problem further, and in particular seek some knowledge of the radial displacements on the inner surface.

Continuing with the development, the inequality (7) provides an upper bound on the potential energy functional, $V_{p}$, associated with the actual displacement field. However, the potential energy does not have a meaning of sufficient significance for values of the functional itself to be of any utility. The form of the functional $V_{p}$ must therefore be modified so that it may be possible to bound a quantity more characteristic of a given problem. The theorem of virtual work provides the following useful relation among quantities at the equilibrium state.

$$
\int_{R} \sigma_{i i} \epsilon_{i j} d R=\int_{S} T_{i} u_{i} d S+\int_{R} F_{i} u_{i} d R .
$$

The use of Eq. (9) allows $V_{p}$ to be expressed as

$$
V_{\nu}=\frac{1}{2} \int_{S u} T_{i} u_{i} d S-\frac{1}{2} \int_{S T} T_{i} u_{i} d S-\frac{1}{2} \int_{R} F_{i} u_{i} d R .
$$

Combining (7) and (10) we have

$$
\frac{1}{2} \int_{S u} T_{i} u_{i} d S-\frac{1}{2} \int_{S T} T_{i} u_{i} d S-\frac{1}{2} \int_{R} F_{i} u_{i} d R \leq V_{p}^{\circ}
$$

where

$$
\begin{aligned}
V_{p}^{\circ}=\frac{1}{2} \int_{R}\left\{\frac{G}{2}\left[\left(\frac{\partial u_{i}^{\circ}}{\partial x_{i}}\right)^{2}+\left(\frac{\partial u_{i}^{\circ}}{\partial x_{i}}\right)^{2}+2 \frac{\partial u_{i}^{\circ}}{\partial x_{i}} \frac{\partial u_{i}^{\circ}}{\partial x_{i}}\right]\right. & \left.+\left(k-\frac{2}{3} G\right) \frac{\partial u_{i}^{\circ}}{\partial x_{l}} \frac{\partial u_{m}^{\circ}}{\partial x_{m}}\right\} d R \\
& -\int_{R} F_{i} u_{i}^{\circ} d R-\int_{S_{\boldsymbol{T}}} T_{i} u_{i}^{\circ} d S .
\end{aligned}
$$

Considering again the heterogeneous sphere, introduction of the conditions of (8) into (11) gives the following inequality:

$$
-\frac{1}{2} \int_{S} p_{A}\left(u_{r}\right)_{A} d S \leq V_{p}^{\circ}
$$

where the integration is carried out over the inner surface, and $\left(u_{r}\right)_{A}$ is the radial displacement on this surface. This later form is greatly simplified, and we note that $\left(u_{r}\right)_{A}$ is now the only random variable which explicitly appears in $V_{p}$.

In general, since $G(x)$ and $k(x)$ may be random functions of position, it follows that $V_{p}$ and $V_{p}^{\circ}$ will have random values, differing for each member of an ensemble of macroscopically similar systems. If both sides of (11) are averaged in an ensemble sense, the result is

$$
\frac{1}{2} \int_{S u} \overline{T_{i} u_{i}} d S-\frac{1}{2} \int_{S T} \overline{T_{i} u_{i}} d S-\frac{1}{2} \int_{R} F_{i} \overline{u_{i}} d R \leq \bar{V}_{p}^{\circ}
$$


where $\bar{V} \circ$ is given by

$$
\begin{aligned}
& \bar{V}_{D}^{\circ}=\frac{1}{2} \int_{R} \overline{\left\{\frac{G}{2}\left[\left(\frac{\partial u_{i}^{\circ}}{\partial x_{i}}\right)^{2}+\left(\frac{\partial u_{i}^{\circ}}{\partial x_{i}}\right)^{2}+2 \frac{\partial u_{i}^{\circ}}{\partial x_{i}} \frac{\partial u_{i}^{\circ}}{\partial x_{i}}\right]\right.}+\overline{\left.\left(k-\frac{2}{3} G\right) \frac{\partial u_{i}^{\circ}}{\partial x_{l}} \frac{\partial u_{m}^{\circ}}{\partial x_{m}}\right\}} d R \\
& -\int_{R} F_{i} \bar{u}_{i}^{\circ} d R-\int_{S_{T}} \overline{T_{i} u_{i}^{\circ}} d S .
\end{aligned}
$$

The ensemble averaging process makes the evaluation of the admissible functional more tractable. Depending upon the choice of admissible functions, $\bar{V}_{p}^{\circ}$ may be evaluated in terms of a limited amount of statistical information, usually no more than low order correlation functions. By contrast the evaluation of $V_{p}^{\circ}$ as expressed in (12) requires total knowledge of the random elastic coefficients for individual physical systems of the ensemble.

In addition to the difficulty in evaluating the bound on a quantity associated with an individual system of the ensemble, the information provided is of value only in the context of similar information for a great many systems. It is perhaps more direct to bound an ensemble average quantity which in an overall statistical sense provides a better measure of the problem.

We note that the fluctuating part of a quantity vanishes in those regions for which the value of the quantity is prescribed. It follows that

$$
\begin{aligned}
& \bar{T}_{i}=T_{i} \text { and } T_{i}^{\prime}=0 \text { on } S_{T}, \\
& \bar{u}_{i}=u_{i} \text { and } u_{i}^{\prime}=0 \text { on } S_{u} .
\end{aligned}
$$

Thus since $T_{i}$ and $u_{i}$ are deterministic on $S_{T}$ and $S_{u}$, respectively, the functional $\bar{V}_{p}$ which appears on the left side of (14) may be expressed as

$$
\bar{V}_{p}=\frac{1}{2} \int_{S u} \bar{T}_{i} u_{i} d S-\frac{1}{2} \int_{S T} T_{i} \bar{u}_{i} d S-\frac{1}{2} \int_{R} F_{i} \bar{u}_{i} d R .
$$

The ensemble average value of $V_{p}$ can thus generally be expressed in terms of products of average quantities. We note, however, that the average of $V_{p}$ as expressed in (6) would involve correlation functions not usually identified with quantities of interest. The form of (16) is considerably more useful for the purposes of this work, as is illustrated by the following expression to which it reduces for the heterogeneous sphere problem:

$$
\bar{V}_{p}=-\frac{1}{2} \int_{S} p_{A}\left(\bar{u}_{r}\right)_{A} d S .
$$

Principle of minimu complementary energy. The functional

$$
V_{c}=\frac{1}{2} \int_{R} \sigma_{i j} \epsilon_{i ;} d R-\int_{S u} T_{i} u_{i} d S
$$

which may be written explicitly in terms of stress as

$$
V_{c}=\frac{1}{2} \int_{R}\left[\frac{\sigma_{i,} \sigma_{i j}}{2 \sigma_{r}}-\frac{1}{6}\left(\frac{1}{G}-\frac{2}{3 k}\right) \sigma_{l \imath} \sigma_{m m}\right] d R-\int_{S u} T_{i} u_{i} d S
$$

and, where the admissible stress fields are symmetric, satisfy the traction boundary conditions, and the subsidiary conditions

$$
\partial \sigma_{i j} / \partial x_{i}+F_{i}=0 \quad \text { in } R,
$$


is stationary and has an absolute minimum for the stress field which satisfies the compatibility relations or, equivalently, which is derivable from a displacement field $u_{i}$ such that

$$
\frac{\sigma_{i j}}{2 G}-\frac{1}{6}\left(\frac{1}{G}-\frac{2}{3 k}\right) \sigma_{l l} \delta_{i i}=\frac{1}{2}\left(\frac{\partial u_{i}}{\partial x_{i}}+\frac{\partial u_{i}}{\partial x_{i}}\right) .
$$

Employing previous notation, we shall denote the extremum value of the functional corresponding to the actual state of stress by $V_{c}$, and the value of the functional associated with any admissible stress field, $\sigma_{i i}^{\circ}$, by $V_{c}^{\circ}$. The complementary energy principle then yields the inequality

$$
V_{c} \leq V_{c}^{\circ} \text {. }
$$

Following a procedure similar to that employed for the potential energy theorem, we utilize the virtual work expression to provide a modified form of $V_{c}$, which appears below:

$$
V_{c}=\frac{1}{2} \int_{S T} T_{i} u_{i} d S-\frac{1}{2} \int_{S u} T_{i} u_{i} d S+\frac{1}{2} \int_{R} F_{i} u_{i} d R .
$$

If we introduce (21) into (20) and for previously expressed reasons take the ensemble average of both sides of (20), we obtain the following result:

$$
\frac{1}{2} \int_{S T} T_{i} \bar{u}_{i} d S-\frac{1}{2} \int_{S u} \bar{T}_{i} u_{i} d S+\frac{1}{2} \int_{R} F_{i} \bar{u}_{i} d R \leq \bar{V}_{c}^{\circ}
$$

where the expression for $\bar{V}_{c}^{\circ}$ is

$$
\bar{V}_{c}^{\circ}=\frac{1}{2} \int_{R}\left[\overline{\frac{\sigma_{i j}^{\circ} \sigma_{i i}^{\circ}}{2} \frac{1}{G}}-\frac{1}{6} \overline{\left(\frac{1}{G}-\frac{2}{3 k}\right) \sigma_{l i}^{\circ} \sigma_{m m}^{\circ}}\right] d R-\int_{S u} \bar{T}_{i}^{\circ} u_{i} d S .
$$

For the particular problem of the heterogeneous sphere, inequality (22) reduces to the form below:

$$
\int_{S} p_{A}\left(\overline{u_{r}}\right)_{A} d S \leq \bar{V}_{c}^{\circ}
$$

Admissible Functions. Referring to (14) and (15), it can be seen that the expression for $\bar{V}_{p}^{\circ}$, which must be evaluated in order to obtain an upper bound on $\bar{V}_{p}$, is in terms of correlations of the elastic coefficients and the admissible displacement field. These correlation functions are generally quite difficult to obtain. We shall therefore limit our choice of admissible functions to those which are statistically independent of the random elastic coefficients. Although the possibility of finding a useful admissible function which has a fluctuating component and which is uncorrelated with $G(x)$ and $k(x)$ cannot be entirely eliminated, it is highly unlikely. In a practical sense, therefore, we are limited to admissible functions with no fluctuating component, henceforth referred to as deterministic admissible functions.

With a deterministic admissible displacement field, the expression for $\bar{V}_{p}^{\circ}$, as given in (15), reduces to the form

$$
\begin{aligned}
\bar{V}_{p}^{\circ}=\frac{1}{2} \int_{R}\left\{\frac{\bar{G}}{2}\left[\left(\frac{\partial u_{i}^{\circ}}{\partial x_{i}}\right)^{2}+\left(\frac{\partial u_{i}^{\circ}}{\partial x_{i}}\right)^{2}+2 \frac{\partial u_{i}^{\circ}}{\partial x_{i}} \frac{\partial u_{i}^{\circ}}{\partial x_{i}}\right]\right. & \left.+\left(\bar{k}-\frac{2}{3} \bar{G}\right) \frac{\partial u_{i}^{\circ}}{\partial x_{l}} \frac{\partial u_{m}^{\circ}}{\partial x_{m}}\right\} d R \\
& -\int_{R} F_{i} u_{i}^{\circ} d R-\int_{S T} T_{i} u_{i}^{\circ} d S .
\end{aligned}
$$


We note that the evaluation of $\bar{V}_{\nu}^{\circ}$ can now be carried out with a knowledge of only $\bar{G}$ and $\bar{k}$.

Although the restriction that admissible functions be deterministic certainly reduces the amount of information necessary for an evaluation of the bound, it must be realized that admissible fields with relevant random components would possibly yield better results. Recent work [6] with statistically homogeneous problems has, in fact, verified that the quality of bounds obtained from classical minimum principles may be improved through the inclusion of more statistical information, specifically higher order correlation functions of material coefficients. It has been found [7], however, that the application of these procedures to problems which are not statistically homogeneous is considerably more complex and requires explicit statistical information, not generally available.

Proceeding with the development, we observe that since $\tilde{V}_{p}^{\circ}$ provides an upper bound on $\bar{V}_{p}$, it is desirable to minimize $\bar{V}_{p}^{\circ}$ for any restricted class of admissible functions which is being used. In particular, we seek the deterministic admissible field which provides the minimum value of $\bar{V}_{p}^{\circ}$. Pursuing this objective, we note that $\bar{V}_{p}^{\circ}$ as expressed in (25) may be identified with the potential energy functional of a fictitious body. This fictitious body is subjected to the same boundary and loading conditions and is identical in all respects to the actual randomly heterogeneous body except that it has elastic coefficients $\bar{G}$ and $\bar{k}$. Utilizing the theorem of minimum potential energy, it follows immediately that this functional assumes its absolute minimum value for the admissible displacement field which corresponds to the equilibrium state of the abovedefined fictitious body. Thus the deterministic admissible displacement field which provides the minimum value of $\bar{V}_{p}^{\circ}$, and hence the best bound on $\bar{V}_{p}$, is the solution of a "reference" problem completely analogous to the actual problem but with elastic properties $\bar{G}$ and $\bar{k}$. The quantitics associated with the solution of this reference problem will be denoted by an $\left(^{*}\right)$. For this choice of an admissible field, utilization of the virtual work theorem allows modification of the form of $\bar{V}_{n}^{\circ}$ from that of (25) to the expression which appears below:

$$
\bar{V}_{p}^{\circ}=\frac{1}{2} \int_{S u} T_{i}^{*} u_{i} d S-\frac{1}{2} \int_{S T} T_{i} u_{i}^{*} d S-\frac{1}{2} \int_{R} F_{i} u_{i}^{*} d R .
$$

The inequality of (14) may then be written as

$$
\begin{aligned}
& {\left[\frac{1}{2} \int_{S u} \bar{T}_{i} u_{i} d S-\frac{1}{2} \int_{S_{T}} T_{i} \bar{u}_{i} d S-\frac{1}{2} \int_{R} F_{i} \bar{u}_{i} d R\right]} \\
& \leq\left[\frac{1}{2} \int_{S u} T_{i}^{*} u_{i} d S-\frac{1}{2} \int_{S_{T}} T_{i} u_{i}^{*} d S-\frac{1}{2} \int_{R} F_{i} u_{i}^{*} d R\right] .
\end{aligned}
$$

We next consider evaluation of the bound provided by the complementary energy principle. For reasons analogous to those stated above, admissible functions are again restricted to be deterministic. $\bar{V}_{0}^{\circ}$ then reduces to

$$
\bar{V}_{c}^{\circ}=\frac{1}{2} \int_{R}\left\{\frac{\sigma_{i,}^{\circ} \sigma_{i, j}^{\circ}}{2} \overline{\left(\frac{1}{(i}\right)}-\frac{1}{6}\left[\overline{\left(\begin{array}{c}
1 \\
C_{i}
\end{array}\right)}-\frac{2}{\overline{3}} \overline{\left(\frac{1}{k}\right)}\right] \sigma_{\imath i \sigma_{m m}^{\circ}}^{\circ}\right\} d R-\int_{S_{u}} T_{i}^{\circ} u_{i} d S .
$$

$\bar{V}_{c}^{\circ}$ provides an upper bound on $\bar{l}_{c}$. It follows that as $\bar{V}_{c}^{\circ}$ decreases, the quality of the bound improves. We observe that expression (28) is the complementary energy 
functional associated with a fictitious body identical to the actual body but with elastic coefficients

$$
\left[\left(\frac{1}{G}\right)\right]^{-1} \text { and }\left[\left(\frac{1}{k}\right)\right]^{-1}
$$

It then follows directly from the theory of minimum complementary energy that of all deterministic admissible stress fields, the one which provides the absolute minimum value of $\bar{V}_{c}^{\circ}$ for this restricted class of admissible functions is the solution of a "reference" problem completely analogous to the actual problem but with elastic coefficients

$$
\left[\left(\frac{1}{G}\right)\right]^{-1} \text { and }\left[\left(\frac{1}{k}\right)\right]^{-1} \text {. }
$$

We denote the quantities associated with the solution of this reference problem by $\left.{ }^{* *}\right)$ superscript. The introduction of this admissible field into $\bar{V}_{c}^{\circ}$ and utilization of the virtual work expression finally allows the inequality of (22) to be written in the form

$$
\begin{aligned}
& {\left[\frac{1}{2} \int_{S_{T}} T_{i} \bar{u}_{i} d S-\frac{1}{2} \int_{S_{u}} \bar{T}_{i} u_{i} d S+\frac{1}{2} \int_{R} F_{i} \bar{u}_{i} d R\right]} \\
& \leq\left[\frac{1}{2} \int_{S_{T}} T_{i} u_{i}^{* *} d S-\frac{1}{2} \int_{S_{u}} T_{i}^{* *} u_{i} d S+\frac{1}{2} \int_{R} F_{i} u_{i}^{* *} d R\right] .
\end{aligned}
$$

The above discussion of admissible functions can be applied directly to the heterogeneous sphere problem. Recalling the values of $\bar{V}_{p}$ and $\bar{V}_{c}$ which were previously obtained for this problem, the general inequalities (27) and (29) provide the following lower and upper bounds, respectively:

$$
p_{A} \int_{S}\left(u_{r}^{*}\right)_{A} d S \leq p_{A} \int_{S}\left(\overline{u_{r}}\right)_{A} d S \leq p_{A} \int_{S}\left(u_{r}^{* *}\right)_{A} d S .
$$

We assume now that the distribution of material properties within the sphere is statistically homogeneous and isotropic. The macroscopic symmetry of the configuration then allows that $(\overline{u r})_{A}$, the ensemble average radial displacement at the inner surface, and the analogous quantities associated with the reference problems are independent of angular variation. This macroscopic symmetry permits the further reduction of (30) to the following bounds on $\left(\overline{u_{r}}\right)_{A}$ :

$$
\left(u_{r}^{*}\right)_{A} \leq\left(\overline{u_{r}}\right)_{A} \leq\left(u_{r}^{* *}\right)_{A} .
$$

Introducing expressions for $\left(u_{r}^{*}\right)_{A}$ and $\left(u_{r}^{* *}\right)_{A}$, the bounds take the final form given below:

$$
\begin{aligned}
\frac{p_{A} A\left(2 A^{3}+B^{3}\right)}{12\left(B^{3}-A^{3}\right)}\left[\left(\frac{1}{G}\right)\right. & \left.+4\left(\frac{1}{k}\right)\right]+\frac{p_{A} A}{6}\left[\left(\frac{1}{G}\right)-\frac{2}{3}\left(\frac{1}{k}\right)\right] \\
\leq & \left(\bar{u}_{r}\right)_{A} \leq \frac{p_{1} A\left(2 A^{3}+B^{3}\right)}{12\left(B^{3}-A^{3}\right)}\left[\frac{1}{\bar{G}}+\frac{4}{\bar{k}}\right]+\frac{p_{A} A}{6}\left[\frac{1}{\bar{G}}-\frac{2}{3 \bar{k}}\right] .
\end{aligned}
$$

While the above bounds on the ensemble average radial displacement certainly do not comprise a complete solution, they do provide some information about a quantity characteristic of the problem.

In general, the problems for which the inequalities of (25) and (29) may provide 
useful results are those for which the geometrical boundaries and prescribed conditions allow a quantity of interest to be isolated in the functional expressions. For those problems in which these conditions are fulfilled, final results follow directlı upon the introduction of the proper admissible functions.

An illustration of this statement is provided by the following problem:

Heterogeneous elastic cylinder under uniform axial load. We consider a randomly heterogeneous elastic cylinder of cross section $S_{0}$ and length $2 L$. The axis of the cylinder is taken to be in the $x_{3}$ direction of a coordinate system with origin at the geometric centroid. Uniformly distributed tensile forces of intensity $\sigma_{n}$ act on the end faces of the cylinder. The traction on the other cylinder surfaces and the body forces are assumed to be zero. Boundary conditions for this problem can then be written as follows:

(a) $T_{3}=\sigma_{0}$ at $X_{3}=L, \quad T_{3}=-\sigma_{0}$ at $X_{3}=-L$.

(b) All other components of the traction vector are identically zero over the entire surface.

We now assume the random distribution of elastic properties to be statistically homogeneous and isotropic, and the body to be large compared to a characteristic length of the inhomogeneities. Combining this assumption with conditions (a) and (b), we would expect the ensemble average displacements to be uniform over the end faces. The inequality of (27) then reduces to

$$
-\left[\left(\bar{u}_{3}\right)_{X_{3}-L}-\left(\bar{u}_{3}\right)_{X_{3}=-L}\right] \sigma_{0} S_{0} \leq-\left[\left(u_{3}^{*}\right)_{X_{3}=L}-\left(u_{3}^{*}\right)_{X_{3}=-L}\right] \sigma_{0} S_{0} .
$$

The macroscopic symmetry of the problem with respect to the $X_{3}=0$ plane indicates $\left(\bar{u}_{3}\right)_{X_{2}-L}$ is equal to $-\left(\bar{u}_{3}\right)_{X_{3--L}}$, and $\left(u_{3}^{*}\right)_{X_{3}-L}$ is equal to $-\left(u_{3}^{*}\right)_{X_{3}}={ }_{-L}$. Relation (33) then provides a lower bound on the ensemble average value of the elongation, actually one half the elongation, $\left(\bar{u}_{3}\right)_{X_{0}=L}$ :

$$
\left(\bar{u}_{3}\right)_{x_{3-L}} \geq\left(u_{3}^{*}\right)_{x_{0}-L}
$$

Similarly the inequality of (29) gives an upper bound

$$
\left(\bar{u}_{3}\right)_{X_{3}=L} \leq\left(u_{3}^{* *}\right)_{X_{3}=L} .
$$

Combining (34) and (35) with the easily obtained expressions for $\left(u_{3}^{*}\right)_{x_{0}=L}$ and $\left(u_{3}^{* *}\right)_{x_{3-L}}$ gives the following result:

$$
\sigma_{0} L\left[\frac{3 \bar{k}+\bar{G}}{9 \bar{k} \bar{G}}\right] \leq\left(\bar{u}_{3}\right)_{X_{3}=L} \leq \sigma_{0} L \frac{3\left[\left(\frac{1}{k}\right)\right]^{-1}+\left[\left(\frac{1}{C_{i}}\right)\right]^{-1}}{9\left[\left(\frac{1}{l_{i}}\right)\right]^{-1}\left[\left(\begin{array}{c}
1 \\
(i
\end{array}\right)\right]^{-1}} \mid
$$

It is noted that no assumptions concerning the statistical homogeneity of the stress and strain fields were made in the general develepment leading $t,(27)$ and (29). However, it seems very reasonable due to the particular geometry and prescribed conditions of this problem that the ensemble arerage stress and strain fiells are homogeneous. It may be shown that the volume average value of $\sigma_{33}$ is equal $t$ o $\sigma_{10}$, while the volume average of all other stress components is zero. These are alio the encemble average values of the stress components which can reasonably be expected. We can then assume that ensemble average and volume average stresses are interchangeable for this problem. 
If an effective Young's modulus, $(E)_{\text {eff }}$, is defined for the heterogeneous material, it provides the following relation:

$$
\epsilon_{0}=\sigma_{0} /(E)_{\mathrm{eff}}
$$

where $\epsilon_{0}$ is the uniform value of $\bar{\epsilon}_{33}$. But $\epsilon_{0}$ is just equal to $\left(\bar{u}_{3}\right)_{X_{3}=L} / L$, so

$$
\left(\bar{u}_{3}\right)_{X_{0}=L}=\sigma_{0} L /(E)_{\mathrm{er} \ell} \text {. }
$$

The introduction of (37) into (36) yields bounds on $(E)_{\text {eff }}$

$$
\frac{9\left[\left(\frac{1}{k}\right)\right]^{-1}\left[\left(\frac{1}{G}\right)\right]^{-1}}{3\left[\left(\frac{1}{k}\right)\right]^{-1}+\left[\left(\frac{1}{G}\right)\right]^{-1}} \leq(E)_{\mathrm{eff}} \leq \frac{9 \bar{k} \bar{G}}{3 \bar{k}+\bar{G}} .
$$

The bounds of (38) are the same as those obtained by Paul [1] also through the use of classical variational principles, but in a manner somewhat different in detail than the approach used presently. They are derived here to illustrate that where the concept of an effective property is applicable, previous results can be regained.

Classical variational principles have in the past been used to obtain elementary bounds on effective properties relevant to several physical areas. The formulation of the problems was always based upon the assumption of statistical homogeneity of the random material properties and the physical fields. Reference to the previous work would indicate that the admissible functions used in the derivation of the elementary bounds were deterministic and associated with homogeneous fields. These admissible functions were, in the context of the present paper, the solutions of the relevant reference problems, analogous to those defined above. It follows from the discussion accompanying the definition of the reference problems that the elementary bounds on effective properties are the best which can be found through the use of deterministic admissible functions in classical variational principles. The quality or closeness of the bounds derived with the methods of this paper is of the same order as for the elementary bounds on effective properties. They are of numerical value only when the dispersion of the random coefficients about their mean values is relatively small.

3. Elastically supported beams-governing equations. The theory governing elastically supported beams and by direct mathematical analogy thin axisymmetric circular shells is well developed and widely known. The derivation of these problems from more general considerations points out the limitations and restrictions of the classical "strength of materials" approach which will serve the needs of this paper.

We consider an elastically supported beam, extending from $X=0$ to $L$, for which the elastic support is provided by a load-bearing foundation distributed along the beam length. There may also be additional supports at the beam ends. The beam is loaded by transverse forces which cause the beam to deflect. In turn, these deflections produce vertical reaction forces in the supporting medium which oppose the deflections. It is assumed that the intensity of reaction forces at any point is proportional to the doflection at that point.

The equilibrium of vertical forces and bending moments acting on a beam element of infinitesimal length gives the following equation

$$
d^{2} M / d x^{2}-k w=-p
$$


where

$M(x)$ is the bending moment at a section,

$w(x)$ is the vertical deflection of the neutral axis of the beam,

$p(x)$ is the deterministic applied load per unit length,

$k(x)=b m$, is the foundation stiffness when $b$ is the beam width at section $x$ and $m_{f}$ is the foundation modulus which has dimensions of force per unit area per unit length.

Deflection and applied loads are taken as positive downward. A positive moment puts compression in the upper surface. The shear force $V=d M / d x$ is positive downward on the right face of an element and positive upward on the left face.

The moment curvature relation gives

$$
M=-E I d^{2} w / d x^{2}
$$

which may be equivalently rewritten as

$$
M / E I+d^{2} u / d x^{2}=0 .
$$

$E$ is the Young's modulus of the beam material and $I$ is the moment of inertia of a section about its neutral axis. The introduction of (40) into (39) gives the equilibrium equation in terms of displacement:

$$
\frac{d^{2}}{d x^{\overline{2}}}\left(E I \frac{d^{2} w}{d x^{2}}\right)+k w=p .
$$

In the present work both $E I$ and $k$ may be random functions of position along the beam, with the restriction that variations be consistent with the generally accepted limitations imposed by classical beam theory. Thus in this problem random parameters of both a constitutive and nonconstitutive nature occur. The parameter EI appears in equation (40), which is essentially a constitutive relation, while the nonconstitutive parameter $k$ appears only in the equilibrium equation.

The problem definition is completed by the following boundary conditions:

$$
\text { either } M \text { or } d w / d x \text { must be prescribed at } X=0 \text { and } L \text {, and }
$$$$
\text { cither } Y \text { or } a \text { must be prescribed at } X=0 \text { and } I \text {. }
$$

The deflection, slope, moment, and shear force are continuous along the beam. An exception is the shear force at sections where point loads are applied. However, even there it may be realistically assumed, if necessary, that the load actually acts over a finite distance, thus preserving the continuity of the shear force.

Variatonal Princoples. We will utilize two variational principles associated with the beam problem. The first is a variational principle for which the deflection $w$ is the variational rariable and the equilibrium equation (41) is the extremum condition. This pinciple, which is esentially the principle of minimum potential energy, may be stated as follows.

The functional

$$
\zeta_{w}=\int_{0}^{L}\left[\frac{E l}{2}\left(\frac{d^{2} u}{d x^{2}}\right)^{2}+\frac{l}{2}(u)^{2}-p w\right] d x+\left.M \frac{d w}{d x}\right|_{(0)} ^{(L)}-\left.V w\right|_{(0)} ^{(L)}
$$

is stationary and an absolute minimum for the admissible deflection function which satisfies the equilibrium equation and all boundary conditions of the problem. 
The ( ) notation on a boundary term of $U_{w}$ indicates that the term actually appears at a point only if $M$ or $V$ is prescribed at the point. Thus $M d w / d x$ appears at $X=L$ only when $M I$ is prescribed at this point.

A straightforward application of variational methods allows for the demonstration of the above principle. It is shown that satisfaction of the equilibrium equation and boundary conditions is necessary in order that the first variation of $U_{w}$ may vanish. Functions admissible to the variational principle are required to be continuous and satisfy the kinematic boundary conditions. An admissible function, $w^{\circ}$, may be expressed in the form $w^{\circ}=w+\Delta w$ where $w$ is the actual deflection and $\Delta w$ any continuous function which vanishes where deflection is specified and which has zero derivative where the slope is specified. We introduce such an admissible displacement into the functional associated with this principle. Then using integration by parts, the extremum condition, and the innate positive character of $E I$ and $k$, it can be shown that the extremum value of the functional is the absolute minimum value for any admissible function. This yields the inequality

$$
U_{w} \leq U_{w}^{\circ}
$$

where $U_{w}$ now denotes the extremum value of the functional, and $U_{w}^{\circ}$ the value associated with any admissible function, $w^{\circ}$.

If the above minimum principle is to be useful in the present work, the form of $U_{w}$, as expressed in (41), must be modified. We seek a form more favorable to the isolation of quantities of interest in particular problems.

Using integration by parts, equation (41), and the following relations:

$$
M=-E I \frac{d^{2} w}{d x^{2}} \text { and } V=-\frac{d}{d x}\left(E I \frac{d^{2} w}{d x^{2}}\right),
$$

the functional $U_{w}$ may be rewritten equivalently as

$$
U_{w}=-\frac{1}{2} \int_{0}^{L} p w d x-\left.\frac{1}{2} M \frac{d w}{d x}\right|_{0} ^{L}+\left.M \frac{d w}{d x}\right|_{(0)} ^{(L)}+\left.\frac{1}{2} V w\right|_{0} ^{L}-\left.V w\right|_{(0)} ^{(L)} .
$$

If the ensemble average of (44) is taken, the result is

$$
\bar{U}_{w} \leq \bar{U}_{w}^{\circ} \text {. }
$$

In order to evaluate the bound on $\bar{U}_{w}$, we must evaluate

$$
\bar{U}_{w}^{\circ}=\int_{0}^{L}\left[\overline{\frac{E I}{2}\left(\frac{d^{2} w^{\circ}}{d x^{2}}\right)^{2}}+\overline{\frac{k}{2}\left(w^{\circ}\right)^{2}}-p \tilde{w}^{\circ}\right] d x+\left.M \frac{d \bar{w}^{\circ}}{d x}\right|_{(\cap)} ^{(L)}-\left.V \bar{w}^{\circ}\right|_{(0)} ^{(L)} .
$$

The above functional can be evaluated in terms of the average values of $E I$ and $k$ only for those admissible functions which are statistically independent of the random coefficients. Recalling the discussion following expression (24), we see that these admissible functions must in general be deterministic.

It can be demonstrated in a straightforward manner ${ }^{1}$ that the deterministic admissible deflection field which provides the minimum value of $\vec{U}_{w}^{\circ}$ for this restricted class of admissible functions is the solution of a reference problem analogous to the actual problem but with properties $E I$ and $\bar{k}$. Quantitics associated with the solution of this reference problem are denoted by an $\left({ }^{*}\right)$. For the admissible function $u^{*}$, the functional

\footnotetext{
${ }^{1}$ See Appendix A.
} 
$\bar{U}_{\text {w }}^{0}$ may be rewritten in a form similar to expression (45). The inequality of (46) can then be expressed as

$$
\begin{aligned}
& {\left[-\frac{1}{2} \int_{0}^{L} p \bar{w} d x-\left.\frac{1}{2} \bar{M} \frac{d \bar{w}}{d x}\right|_{0} ^{L}+\left.M \frac{d \bar{w}}{d x}\right|_{(0)} ^{(L)}+\left.\frac{1}{2} \bar{V} \bar{w}\right|_{0} ^{L}-\left.V \bar{w}\right|_{(0)} ^{(L)}\right]} \\
& \quad \leq\left[-\frac{1}{2} \int_{0}^{L} p w^{*} d x-\left.\frac{1}{2} M^{*} \frac{d w^{*}}{d x}\right|_{0} ^{L}+\left.M \frac{d w^{*}}{d x}\right|_{(0)} ^{(L)}+\left.\frac{1}{2} V^{*} w^{*}\right|_{0} ^{L}-\left.V w^{*}\right|_{(0)} ^{(L)}\right] .
\end{aligned}
$$

The expression for $\bar{U}_{w}$ which appears on the left side of (48) can, of course, be obtained only because one quantity or the other of each pair of quantities which occurs in the boundary terms is specified at the boundary point. This allows the average of the product to be written as the product of the averages.

We shall now consider a variational principle analogous to the complementary energy principle for which both the bending moment and the deflection are variational variables. It can be shown that the functional defined as

$$
U_{M}=\frac{1}{2} \int_{0}^{L}\left[\frac{M^{2}}{E I}+k w^{2}\right] d x+\left.M \frac{d w}{d x}\right|_{(0)} ^{(L)}-\left.\frac{d M}{d x} w\right|_{(0)} ^{(L)}
$$

is stationary and an absolute minimum for those admissible functions which are related by the constitutive equation

$$
M / E I+d^{2} w / d x^{2}=0 .
$$

All admissible moment and deflection functions, denoted respectively by $M^{\circ}$ and $w^{\circ}$, must satisfy the equilibrium equation, (39), as a subsidiary condition. In addition admissible moment functions must be continuous and satisfy the prescribed boundary conditions on $M$ and $d M / d x=V$.

The notation ( ) on boundary terms associated with $U_{M}$ indicates that the term is actually present only if $w$ or $d w / d x$ is specified at the point.

Denoting the extremum value of the functional by $U_{M}$ and the value of any admissible functions by $U_{M}^{\circ}$, the above minimum principle gives the following inequality:

$$
U_{M} \leq U_{M}^{\circ} \text {. }
$$

Utilization of the extremum condition (40), and subsequent use of integration by parts and the equilibrium equation, allows $U_{M}$ to be expressed as

$$
U_{M}=\frac{1}{2} \int_{0}^{L} p w d x-\left.\frac{1}{2} M \frac{d w}{d x}\right|_{0} ^{L}+\left.M \frac{d w}{d x}\right|_{(0)} ^{(L)}+\left.\frac{1}{2} V w\right|_{0} ^{L}-\left.V w\right|_{(0)} ^{(L)} .
$$

Taking the ensemble average of (50) we have a bound on $\bar{U}_{M}$

$$
\bar{U}_{M} \leq \bar{U}_{M}^{\circ} \text {. }
$$

As $\bar{U}_{M}^{\circ}$ decreases the quality of the bound will improve. For reasons similar to those presented earlier, we choose deterministic admissible moment functions. It can be shown $^{2}$ that the deterministic admissible moment function which provides the minimum value of $\bar{U}_{M}^{\circ}$ for this restricted class of admissible functions is the moment associated with the solution of a problem completely analogous to the actual problem but with properties

See Appendix B. 


$$
\left[\left(\frac{1}{E I}\right)\right]^{-1} \text { and }\left[\left(\frac{1}{k}\right)\right]^{-1} \text {. }
$$

Denoting this particular admissible moment by $M^{* *}$, defined by

(a) $M^{* *}=-\left[\left(\frac{1}{E I}\right)\right]^{-1} \frac{d^{2} w^{* *}}{d x^{2}}, \quad$ where
(b) $\frac{d^{2}}{d x^{2}}\left\{\left[\left(\frac{1}{E I}\right)\right]^{-1} \frac{d^{2} w^{* *}}{d x^{2}}\right\}+\left[\left(\frac{1}{k}\right)\right]^{-1} w^{* *}=p$,

and combining (a) and (b) gives

(c) $\frac{d^{2} M^{* *}}{d x^{2}}-\left[\left(\frac{1}{k}\right)\right]^{-} w^{* *}=-p$.

Admissibility requires that $M^{* *}$ satisfy the boundary conditions where the moment or the shear force are prescribed, while $w^{* *}$ satisties the boundary conditions on $w$ and $d w / d x$.

We note that although the chosen admissible moment, $M^{* *}$, is deterministic, the admissible deflection, $w^{\circ}$, associated with this choice is not deterministic due to the presence of the random parameter $k(x)$ in the subsidiary condition, Eq. (39). The function $w^{* *}$ is not admissible because it does not satisfy the subsidiary condition for the choice, $M^{\circ}=M^{* *}$. Combining (53c) and the subsidiary condition, the admissible deflection function $w^{\circ}$ can be expressed in terms of $w^{* *}$ as

$$
w^{\circ}=\frac{1}{k}\left[\left(\frac{1}{k}\right)\right]^{-1} w^{* *} .
$$

The introduction of the above admissible functions into $\bar{U}_{M}^{\circ}$, followed by some manipulation, allows a modification of the functional form so that the inequality of (52) may finally be written as

$$
\begin{aligned}
& {\left[\frac{1}{2} \int_{0}^{L} p \bar{w} d x-\left.\frac{1}{2} \bar{M} \frac{d \bar{w}}{d x}\right|_{0} ^{L}+\left.\bar{M} \frac{d w}{d x}\right|_{(0)} ^{(L)}+\left.\frac{1}{2} \bar{V} \bar{w}\right|_{0} ^{L}-\left.\bar{V} w\right|_{(0)} ^{(L)}\right]} \\
& \quad \leq\left[\frac{1}{2} \int_{0}^{L} p w^{* *} d x-\left.\frac{1}{2} M^{* *} \frac{d w^{* *}}{d x}\right|_{0} ^{L}+\left.M^{* *} \frac{d w}{d x}\right|_{(0)} ^{(L)}+\left.\frac{1}{2} V^{* *} w^{* *}\right|_{0} ^{L}-\left.V^{* *} w\right|_{(0)} ^{(L)}\right] .
\end{aligned}
$$

Simply supported Beams. Consider a beam on an elastic foundation which is simply supported at $X=0$ and $X=L$ and acted upon at its midpoint by a concentrated force of magnitude $P$.

The following conditions are thus satisfied:

$$
\begin{aligned}
w & =0 \text { at } X=0 \text { and } L, \\
M & =0 \text { at } X=0 \text { and } L, \text { and } \\
p(X) & =P \delta\left(X-I_{1} / 2\right) .
\end{aligned}
$$

Introdueing these curditions into the gcneral bounds (4S) and (51) yields the following lower and upper bounds, on $(\bar{w})_{X_{-L / 2}}$, the average midpoint deflection, respectively:

$$
\left(w^{*}\right)_{x=L / 2} \leq(\bar{w})_{x=L / 2} \leq\left(w^{* *}\right)_{x=L / 2} .
$$


Assuming the random coefficients are distributed in a statistically homogeneous manner along the beam length, the bounds on $(\bar{w})_{x=L / 2}$ may be more explicitly given as

$$
\frac{P \beta}{2 \bar{k}} \frac{\sinh \beta L-\sin \beta I}{\cosh \beta L+\cos \beta L} \leq(\bar{w})_{X=L / 2} \leq \frac{P \gamma}{2(\overline{1 / k})^{-1}} \frac{\sinh \gamma L-\sin \gamma L}{\cosh \gamma L+\cos \gamma L}
$$

where

$$
\beta=\left[\frac{\bar{k}}{4 \overline{E I}}\right]^{1 / 4} \text { and } \gamma=\left[\frac{\left(\frac{1}{k}\right)^{-1}}{4\left(\frac{1}{E I}\right)^{-1}}\right]^{1 / 4}
$$

If the statistics of the random coefficients were not homogeneous, the calculation of the bounds could have proceeded with a knowledge of

$$
\overline{E I(x)}, \overline{k(x)}, \overline{\frac{1}{E I(x)}} \text { and } \overline{\frac{1}{k(x)}} \text {. }
$$

There are other problems of a similar nature for which results can be obtained. These first order bounds may also be combined in a kind of hierarchy to provide bounds on the ensemble average influence coefficient and various correlation functions. A number of the above-mentioned results may be found in reference (7) along with analogous results for elastically supported plates.

\section{Appendix A.}

Demonstration that $w^{*}$ provides minimum Value of $\bar{U}_{w}^{\circ}$ For any Deterministic ADMISSIBLE FUNCTION. The introduction of a deterministic admissible deflection function into the expression for $\bar{U}_{w}^{\circ}$ given in (47) yields

$$
\bar{U}_{\mathrm{o}}^{\circ}=\int_{0}^{L}\left[\frac{E I}{2}\left(\frac{d^{2} w^{\circ}}{d x^{2}}\right)^{2}+\frac{\bar{E}}{2}\left(w^{\circ}\right)^{2}-p w^{\circ}\right] d x+\left.M \frac{d w^{\circ}}{d x}\right|_{(0)} ^{(L)}-\left.V w^{\circ}\right|_{(0)} ^{(L)} .
$$

As previously stated, the particular admissible function denoted by $w^{*}$ is the solution of the "reference" problem presented below:

(a) $\frac{d^{2}}{d x^{2}}\left(\overline{E I} \frac{d^{2} w^{*}}{d x^{2}}\right)+\bar{k} w^{*}=p$

(b) $w^{*}$ satisfies the kinematic boundary conditions,

(c) $M^{*} \equiv \overline{E I} d^{2} w^{*} / d x^{2}$ and $V^{*} \equiv-d / d x\left(\overline{E I} d^{2} w^{*} / d x^{2}\right)$ satisfy the moment and shear force boundary conditions.

We now define a general deterministic admissible function $w^{\circ}$ as

$$
w^{\circ}=w^{*}+\Delta w^{\circ}
$$

where $w^{*}$ is the solution of the problem $(\mathrm{A}-2)$, and $\Delta w^{\circ}$ is an incremental field of arbitrary magnitude. The admissible function $w^{\circ}$ satisfies the following conditions:

(a) $w^{\circ}$ is continuous,

(b) $w^{\circ}$ satisfies the deflection boundary conditions.

The incremental field, $\Delta w^{\circ}$, satisfies:

(a) $\Delta w^{\circ}$ is continuous, 
(b) $\quad \Delta w^{\circ}=0$ at the boundary points where the deflection is specified and $d \Delta w^{\circ} / d x=0$ where $d w / d x$ is specified.

Introducing $w^{\circ}$ from (A-3) into $\bar{U}_{w}^{\circ}$ gives the following expression:

$$
\begin{aligned}
& \bar{U}_{w}^{\circ}=\bar{U}_{w}^{*}+\frac{1}{2} \int_{0}^{L}\left[\overline{E I}\left(\frac{d^{2} \Delta w^{\circ}}{d x^{2}}\right)^{2}+\bar{k}\left(\Delta w^{\circ}\right)^{2}\right] d x \\
& +\int_{0}^{L}\left[\overline{E I} \frac{d^{2} w^{*}}{d x^{2}} \frac{d^{2} \Delta w}{d x^{2}}+\bar{k} w^{*} \Delta w^{\circ}-p \Delta w^{\circ}\right] d x+\left.M \frac{d \Delta w^{\circ}}{d x}\right|_{(0)} ^{(L)}-\left.V \Delta w^{\circ}\right|_{(0)} ^{(L)} .
\end{aligned}
$$

The first term $\bar{U}_{w}^{*}$ is the value of the functional for $w^{*}$. Using integration by parts and the conditions (A-2(a) and (b)) and (A-5 (b)), it may be shown that the last three terms on the right side of $(A-6)$ vanish. Since

$$
\int_{0}^{L}\left[\overline{E I}\left(\frac{d^{2} \Delta w^{\circ}}{d x^{2}}\right)^{2}+\bar{k}\left(\Delta w^{\circ}\right)^{2}\right] d x
$$

is greater than or equal to zero, the following inequality results:

$$
\bar{U}_{w}^{*} \leq \bar{U}_{w}^{\circ} \text {. }
$$

It has thus been shown that the deterministic function which satisfies the "reference" problem defined in (A-2) provides the absolute minimum value of $\bar{U}_{w}^{\circ}$ for any deterministic admissible function.

\section{Appendix B.}

Demonstration that $M^{* *}$ provides the minimum value of $\bar{U}_{M}^{\circ}$ For any deterMINISTIC ADMISSIBLE MOMENT FUNCTION. Given any admissible moment and deflection functions, the functional $U_{M}^{\circ}$ takes the form

$$
U_{\mathrm{M}}^{\circ}=\frac{1}{2} \int_{0}^{L}\left[\frac{\left(M^{\circ}\right)^{2}}{E I}+k\left(w^{\circ}\right)^{2}\right] d x+\left.\frac{d w}{d x} M^{\circ}\right|_{(0)} ^{(L)}-\left.w \frac{d M^{\circ}}{d x}\right|_{(0)} ^{(L)} .
$$

As previously noted, the admissible functions must satisfy the following conditions:

(a) $d^{2} M^{\circ} / d x^{2}-k w^{\circ}=-p$,

(b) $M^{\circ}$ satisfies the prescribed moment boundary conditions,

(c) $d M^{\circ} / d x$ satisfies the prescribed shear force conditions,

(d) $M^{\circ}$ continuous.

Making use of the condition of admissibility, equation (B-2 (a)), we may express $w^{\circ}$ in terms of the admissible moment as

$$
w^{\circ}=\frac{1}{k}\left[p+\frac{d^{2} M^{\circ}}{d x^{2}}\right] .
$$

For deterministic admissible moment functions we may write

$$
M^{\circ}=M^{* *}+\Delta M^{\circ}
$$

where $M^{* *}$ is defined by the reference problem associated with the equations and discussion of (5.3), and $\Delta .11^{\circ}$ is an incremeital deteministic field which is required only to satisfy the following conditions:

(a) $\Delta M^{\circ}=0, \quad$ where the moment is specified, 
(b) $d \Delta M^{\circ} / d x=0, \quad$ where the shear force is specified,

(c) $\Delta M^{\circ}$ continuous.

With the introduction of $M^{\circ}$, as written in (B-4), into (B-3) and the use of expression (53c), we have

$$
w^{\circ}=\frac{1}{k}\left[\left(\frac{1}{k}\right)^{-1} w^{* *}+\frac{d^{2} \Delta M^{\circ}}{d x^{2}}\right]
$$

Thus the ensemble average value of the functional expressed in (B-1) assumes the following form for any deterministic admissible moment function:

$$
\begin{aligned}
\bar{U}_{M}=\frac{1}{2} \int_{0}^{L}[ & \left(M^{* *}\right)^{2}\left(\overline{\frac{1}{E I}}\right)+\left(\Delta M^{\circ}\right)^{2}\left(\overline{\frac{1}{E I}}\right)+2 M^{* *} \Delta M^{\circ}\left(\overline{\frac{1}{E I}}\right) \\
& \left.+\left(\frac{1}{k}\right)^{-1}\left(w^{* *}\right)^{2}+2 w^{* *} \frac{d^{2} \Delta M^{\circ}}{d x^{2}}+\left(\frac{1}{k}\right)\left(\frac{d^{2} \Delta M^{\circ}}{d x^{2}}\right)^{2}\right] d x \\
& +\left.\frac{d w}{d x} M^{* *}\right|_{(())} ^{(L)}+\left.\frac{d w}{d x} \Delta M^{\circ}\right|_{(0)} ^{(L)}-\left.w \frac{d M I^{* *}}{d x}\right|_{(0)} ^{(L)}-\left.w \frac{d \Delta M^{\circ}}{d x}\right|_{(0)} ^{(L)} .
\end{aligned}
$$

Regrouping terms we have

$$
\begin{aligned}
\bar{U}_{M}^{\circ}= & \bar{U}_{M}^{* *}+\frac{1}{2} \int_{0}^{L}\left[\left(\Delta M^{\circ}\right)^{2}\left(\frac{1}{E I}\right)+\left(\frac{d^{2} \Delta M^{\circ}}{d x^{2}}\right)\left(\frac{1}{k}\right)\right] d x \\
& +\int_{0}^{L}\left[M^{* *} \Delta M^{\circ}\left(\frac{1}{E I}\right)+w^{* *}\left(\frac{d^{2} \Delta M^{\circ}}{d x^{2}}\right)\right] d x+\left.\frac{d w}{d x} \Delta M^{\circ}\right|_{(0)} ^{(L)}-\left.w \frac{d \Delta M^{\circ}}{d x}\right|_{(0)} ^{(L)}
\end{aligned}
$$

where $\bar{U}_{M}^{* *}$ is the value of $\bar{U}_{M}^{\circ}$ for the choice $M^{\circ}=M^{* *}$. Using (53a), integration by parts, and the boundary conditions imposed on $w^{* *}$, it may be shown that the terms

$$
\int_{0}^{L}\left[M^{* *} \Delta M^{\circ}\left(\frac{1}{E I}\right)+w^{* *} \frac{d^{2} \Delta M^{\circ}}{d x^{2}}\right] d x+\left.\frac{d w}{d x} \Delta M^{\circ}\right|_{(0)} ^{(L)}-\left.w \frac{d \Delta M^{\circ}}{d x}\right|_{(0)} ^{(L)}
$$

vanish. Then since

$$
\int_{0}^{L}\left[\left(\Delta M^{\circ}\right)^{2}\left(\frac{\overline{1}}{L I I}\right)+\left(\frac{d^{2} \Delta M^{\circ}}{d x^{2}}\right)^{2}\left(\frac{1}{k}\right)\right] d x
$$

is nonnegative, the relation of $(13-8)$ yields the following inequality:

$$
\bar{U}_{M}^{* *} \leq \bar{U}_{M}^{\circ} .
$$

\section{REFERENCES}

[1] B. Paul, Prediction of elastic constunts of multiphase materials, Trans. Met. Soc. AIME, 28, 36 (1960)

[2] W. F. Brown, Dielectric constants, permeabilities and conductivities of random media, Trans. Rheology Soc. $9,357(1965)$

[3] M. Beran, Lse of the variational approarh to determine bounds for the effective bulk modulus in heterogeneous media, (quirt. Appl. Math., 24, 107 (1966)

[4] Z. Hashin and S. Shtrihm:n, On some variational principles in anisotropic and nonhomogeneous elasticity, J. Mech. Phys. solid 10, 395: (1962)

[5] I. Sokolnikoff, Mathematical therry uf dusticit!, McC(iraw-IIill, New York, 1956

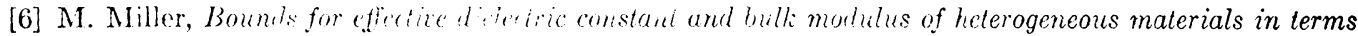
of statistical information, Ph. I). di-iertation, University of Pennsylvania, Philadelphia, Pa., 1967

[7] A. Somoruff, An application of uriational principles to statistically nonhomogeneous fields, Ph.D. dissertation, University of Penns lvania, Philadehphis, Pa., 1968 